New Drug Update

\title{
Lefamulin: novel pleuromutilin drug for community acquired bacterial pneumonia
}

\author{
A. P. Dubey ${ }^{1}$, Sachin Maggo ${ }^{2}$, Awanish $\operatorname{Karan}^{3}$, \\ N. K. Singh ${ }^{4}$, Sreya Bhaskaran ${ }^{5}$
}

${ }^{1}$ Department of Medicine, Santhosh Medical College, Ghaziabad, Uttar Pradesh, India ${ }^{2}$ Department of Medicine, ${ }^{3}$ Department of ENT, Government Hospital, Joshimath, Uttarakhand, India ${ }^{4}$ Department of Medicine, Government Hospital, Tejpur, Assam, India

${ }^{5}$ Department of Medicine, Government Hospital, Roorkee, Uttarakhand, India

Received: 16 October 2019 Accepted: 18 November 2019

\section{*Correspondence to: \\ Dr. Sachin Maggo, Email: sachinmaggo2003@ gmail.com}

Copyright: (C) the author(s), publisher and licensee Medip Academy. This is an openaccess article distributed under the terms of the Creative Commons Attribution NonCommercial License, which permits unrestricted noncommercial use, distribution, and reproduction in any medium, provided the original work is properly cited.

\begin{abstract}
The advent and spread of antimicrobial resistance has led to a global public health emergency necessitating development of new antimicrobial drugs. Community acquired bacterial pneumonia (CABP) contributes a major portion of societal burden with increasing morbidity due to evolution of drug resistant strains. Lefamulin is a novel pleuromutilin antibiotic with unique mechanism of action through inhibition of protein synthesis by binding to the peptidyl transferase center of the 50s bacterial ribosome. The drug displays activity against Gram positive and atypical organisms associated with CABP (i.e., Streptococcus pneumoniae, Haemophilus influenzae, Mycoplasma pneumonia, Legionella pneumophila, and Chlamydophila pneumoniae), with an expanded Gram-positive spectrum including Staphylococcus aureus (i.e., methicillinresistant, vancomycin-intermediate, and heterogeneous strains). Lefamulin is available in both intravenous (IV) and per oral (PO) formulation, exhibits high nonlinear plasma protein binding with low unbound concentrations, higher concentrations in lung epithelial lining fluid (ELF) than in plasma, and a halflife of approximately 10 hour. The recommended IV dose is $150 \mathrm{mg}$ twice daily over 1 hour or a PO dose of $600 \mathrm{mg}$ twice daily. Most common adverse drug reactions injection site reactions, hepatic enzyme elevation, nausea, diarrhoea, hypokalemia, insomnia, and headache. Clinical trials for lefamulin have been positive and Phase 3 data suggest similar efficacy when compared to moxifloxacin with or without linezolid in CABP. Also, the documented resistance and cross-resistance with other Gram-positive antibacterials remains low. With Nabrivia Pharmaceuticals having already received US FDA approval in August 2019, lefamulin may soon be a new addition to the mounting armoury of drugs against $\mathrm{CABP}$.
\end{abstract}

Keywords: Lefamulin, Pleuromutilin, CABP, Multidrug resistance, Gram positive pathogens

\section{INTRODUCTION}

The increasing menace of the post-antibiotic era has incited research and development of new antibacterial agents, however, limited success has been achieved in overall synthesis and discovery of novel antibiotics with unique mechanisms of action. ${ }^{1,2}$ This can be further corroborated by the fact that only 8 new antibacterial agents were Food and Drug Administration (FDA) approved from 2010 to 2018, all of which were derived from well-known drug classes. ${ }^{3}$ In the current scenario of antibacterial development, drugs targeting lethal multidrug-resistant (MDR) Gram-negative bacteria seems promising, in comparison to more common MDR Grampositive bacteria and/or organisms causing sexually transmitted infections. ${ }^{4}$ The later subgroup includes 
momentous burden of community-acquired pneumonia (CAP), with annual costs approximately $€ 10.1$ billion in Europe and over $\$ 17$ billion in the United States. ${ }^{5,6}$ The mortality rate among outpatients is usually $\leq 1 \%$, whereas among hospitalized patients the rate can range from $\sim 12$ to $40 \%$ depending upon the standard of care received. ${ }^{7}$

Risk factors for CAP include alcoholism, asthma, immunosuppression, institutionalization, and an age of $\geq 70$ years. The spectrum of illness generally presents with fever with chills, tachycardia, cough with either nonproductive or productive of mucoid, purulent, or bloodtinged sputum. If the pleura are involved, the patient may experience pleuritic chest pain. The extensive list of potential etiologic agents in CAP includes bacteria, fungi, viruses, and protozoa. Most cases of CAP, however, are caused by relatively few pathogens. The most commonly isolated CAP bacterial pathogen is Streptococcus pneumoniae. Other common causes of communityacquired bacterial pneumonia (CABP) include Haemophilus influenzae, Moraxella catarrhalis, and Staphylococcus aureus. ${ }^{8}$ In view of the rampant rise of antibacterial resistance and limited resources in the armamentarium, novel therapies for CABP are warranted. ${ }^{9-12}$

Lefamulin (formerly known as BC-3781) is a novel, semisynthetic pleuromutilin developed for oral and intravenous administration. The drug impedes prokaryotic ribosomal protein synthesis by binding to the peptidyl transferase centre of the 50S subunit of the bacterial ribosome. ${ }^{13}$ Lefamulin selectively inhibits bacterial ribosomal translation but does not affect eukaryotic ribosomal translation. This unique mechanism of action has been associated with a low probability of cross-resistance to other antimicrobial classes based on in vitro studies. ${ }^{14-16}$ Lefamulin exhibits potent in vitro antibacterial activity against important respiratory pathogens, including Gram-positive species, such as Streptococcus pneumoniae and $S$. aureus (methicillinsusceptible and methicillin-resistant isolates); the fastidious Gram-negative organisms Haemophilus influenzae and Moraxella catarrhalis; and the atypical pathogens Mycoplasma pneumoniae, Chlamydophila pneumoniae and Legionella pneumophila. ${ }^{17}$ The new drug has the advantage of being available in both oral and intravenous (IV) formulations. The US FDA in August 2019 approved Nabriva's Lefamulin (Xenleta) for the treatment of CABP. The novel drug has proven to be a milestone in the treatment of CABP in initial results promising to cater significant unmet needs of both the physicians and the society.

\section{MECHANISM OF ACTION}

Lefamulin is a novel semi-synthetic pleuromutilin [Figure 1]. The chemical structure has a tricyclic mutilin core that is crucial for antimicrobial activity due to the interaction with the central part of the $23 \mathrm{~S}$ rRNA through hydrophobic interactions, van der Waal forces, and hydrogen bonds. ${ }^{16,17}$ The $\mathrm{C}$ (14) side chain is the unique feature responsible for pharmacodynamic and antimicrobial properties of lefamulin. This side chain consists of a thioether bond, which causes increased solubility and metabolic stability, enabling the drug to be administered through both IV and PO routes. ${ }^{17}$ The $\mathrm{C}$ (14) side chain also helps lefamulin overcome bacterial ribosomal mutations and resistance development by maximizing the number of hydrogen bonds to the target site. $^{18}$

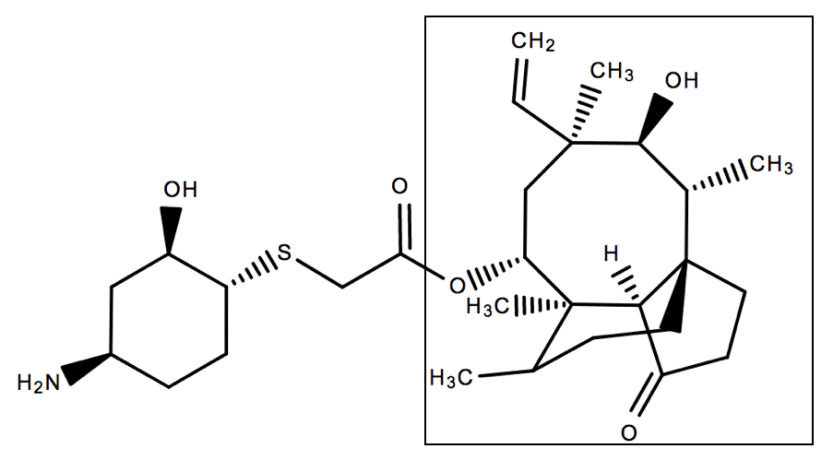

Figure 1: Chemical structure of lefamulin (BC-3781). The tricyclic mutilin core of lefamulin consists of a C21 keto group and various $\mathrm{C} 14$ extensions, which are primarily responsible for the drug's novel mechanism of 50S ribosomal protein synthesis inhibition via the A- and P-sites. ${ }^{16,17}$

Lefamulin inhibits bacterial protein synthesis by interfering with the peptidyl transferase centre by way of binding at the A- and P-site of the 50S ribosome, thereby preventing peptide bond formation. ${ }^{18,19}$ Lefamulin acts via a unique induced-fit mechanism to close the binding pocket within the ribosome, thus enabling tight binding of the drug to the target site. This is a novel mechanism for inhibiting bacterial peptide chain elongation, particularly with the first peptide bond formation, however, once elongation has started, lefamulin is ineffective. Lefamulin selectively inhibits bacterial ribosomal translation but does not affect eukaryotic ribosomal translation. ${ }^{20}$

\section{SPECTRUM OF ACTIVITY}

\section{Gram-positive aerobes}

Lefamulin demonstrates activity against all aerobic Gram-positive organisms except E. faecalis. ${ }^{21,22}$ Also, the drug is effective against resistant strains like methicillinresistant Staphylococcus aureus (MRSA), vancomycinintermediate $S$. aureus (VISA), heterogeneous VISA (hVISA), vancomycin-resistant $S$. aureus and penicillinresistant Streptococcus pneumoniae. ${ }^{21,23}$ Lefamulin also retains susceptibility against macrolide and fluoroquinolone resistant strains of $S$. pneumoniae. ${ }^{24}$ Table 1 compares the minimum inhibitory concentration (MIC) ranges for lefamulin against commonly encountered Gram-positive pathogens. 


\section{Gram-negative aerobes}

Notably, lefamulin has no activity against Pseudomonas aeruginosa, Acinetobacter baumannii, or Enterobacterales due to the presence of the AcrAB-TolC efflux pumps. However, the drug is active against fastidious Gram-negative organisms, including $H$. influenzae and $M$. catarrhalis. $^{21-24}$ Table 1 compares the MIC ranges for lefamulin against commonly used Gramnegative agents.

\section{Anaerobic and atypical organisms}

Lefamulin has demonstrated activity against atypical organisms commonly responsible for CABP, including Chlamydophila spp., Mycoplasma spp., and Legionella spp. (Table 1). While lefamulin has some anaerobic activity, including Clostridium perfringens, Cutibacterium acnes, Fusobacterium spp., Peptostreptococcus spp., Prevotella spp., and Porphyromonas spp., it has weak activity against Bacteroides fragilis and does not cover Clostridioides difficile. ${ }^{21-24}$

\section{CLINICAL PHARMACOLOGY}

Pharmacokinetic and pharmacodynamic assessments are vital parts of drug development programmes for antimicrobial drugs. The pharmacokinetic profile of lefamulin has been studied in a number of pre-clinical and clinical trials. Data obtained from 12 healthy adult subjects following a single-dose of IV lefamulin $150 \mathrm{mg}$ showed the time to maximum serum concentrations (tmax) was $\sim 1$ hour, with a terminal half-life $\left(\mathrm{t}_{1 / 2}\right)$ of about 9-12 hours and a multiphasic decline. ${ }^{25}$ Supplementary studies have shown that the free plasma area under the concentration-time curve over 24 hours ( $f$ AUC0-24) was $1500.8 \mathrm{mgh} / \mathrm{l}$, and the free plasma maximum concentration (Cmax) was $330.1 \mathrm{mg} / 1,22$ given estimated $80-87 \%$ plasma protein binding. ${ }^{26,27}$

The pharmacokinetic properties of the PO lefamulin have also been studied. Oral lefamulin was well-tolerated, and the pharmacokinetics were similar to IV lefamulin 150 $\mathrm{mg}$, but patients in the fed state had reductions in AUCOinf $(10 \%)$ and $\mathrm{Cmax}(28 \%){ }^{28}$ An additional study confirmed PO immediate-release lefamulin $600 \mathrm{mg}$ to have similar pharmacokinetics to IV lefamulin $150 \mathrm{mg}$, with an absolute oral bioavailability of $25.8 \%$ in the fasted state and $21.0 \%$ in the fed state. ${ }^{29}$

Mean plasma protein binding of lefamulin ranges from $94.8 \%$ at $2.35 \mu \mathrm{g} / \mathrm{ml}$ to $97.1 \%$ at $0.25 \mu \mathrm{g} / \mathrm{ml}$ in healthy adults. The mean ( $\min$ to max) steady state volume of distribution of lefamulin is 86.11 (34.2 to 153 1) in patients with CABP after administration of the drug. Following a single IV administration of lefamulin $150 \mathrm{mg}$ to healthy subjects, the highest lefamulin epithelial lining fluid (ELF) concentrations were observed at the end of infusion. The mean ELF and plasma AUC0-8 was 3.87 $\mu \mathrm{g} / \mathrm{ml}$ and $5.27 \mu \mathrm{g} / \mathrm{ml}$, respectively. The estimated ratio of ELF AUC to unbound plasma AUC is approximately $15^{30}$

The mean (min to max) total body clearance of lefamulin is $11.9 \mathrm{l} / \mathrm{h}(2.94$ to $30.0 \mathrm{l} / \mathrm{h})$ in patients with CABP after IV administration of the drug. The mean ( $\min$ to $\max$ ) elimination half-life of lefamulin is approximately 8 hours (3 to 20 hours) in patients with CABP. Lefamulin is primarily metabolized by CYP $3 \mathrm{~A} 4 .{ }^{31}$

Intravenous lefamulin requires dose adjustment in severe hepatic impairment (Child-Pugh Class C), specifically by extending the dosing interval. Oral lefamulin has not been studied and is therefore not recommended in those patients with moderate or severe liver impairment (Child-Pugh Classes B and C). Dosage adjustment for renal impairment, including patients on hemodialysis, is not required as lefamulin predominantly utilizes the hepatobiliary pathway for excretion $(15.5 \%$ and $5.3 \%$ unchanged in urine after $150 \mathrm{mg}$ IV and $600 \mathrm{mg}$ oral dose, respectively). ${ }^{32}$

\section{DOSAGE, DRUG INTERACTIONS AND SPECIAL POPULATION}

Lefamulin can be administered either as $150 \mathrm{mg}$ every 12 hour by intravenous infusion (IV) over $60 \mathrm{~min}$ for 5 to 7 days, or as $600 \mathrm{mg}$ orally every 12 hour for 5 days. $^{32}$

As described above, lefamulin is primarily metabolized by CYP3A4, strong CYP3A inducers like oral rifampin reduce the mean lefamulin AUC0-inf by $28 \%$ and $72 \%$, when administered concomitantly with the injectable and oral formulation of the drug respectively. Likewise, CYP3A inhibitors like oral ketoconazole increase the mean lefamulin AUC0-inf by $31 \%$ and $165 \%$, when administered concomitantly with lefamulin injection and tablets respectively. ${ }^{33}$

No clinically significant differences in the pharmacokinetics of lefamulin were observed based on age, sex, race, weight, or renal impairment including patients receiving hemodialysis. However, in patients with severe hepatic impairment (Child-Pugh Class C), intravenous lefamulin requires dose adjustment, specifically by extending the dosing interval $(150 \mathrm{mg}$ every $24 \mathrm{~h}$ ). Oral lefamulin has not been studied and is therefore not recommended in those patients with moderate or severe liver impairment (Child-Pugh Classes $\mathrm{B}$ and $\mathrm{C}){ }^{34}$

\section{CLINICAL TRIALS}

Two phase III studies lefamulin evaluation against pneumonia (LEAP1 and LEAP2 trials) have established the safety and efficacy of lefamulin in adult patients with CABP. ${ }^{35,36}$ LEAP1 (NCT02559310) was a multicenter, double-blind randomized trial conducted in adult patients with CABP and a Pneumonia Outcomes Research Team 
(PORT) risk class >III. ${ }^{35}$ LEAP1 study patients were randomized $1: 1$ to receive either lefamulin $150 \mathrm{mg}$ IV every $12 \mathrm{~h}$ or moxifloxacin $400 \mathrm{mg}$ IV every $24 \mathrm{~h}$ combined with adjunctive linezolid for any patient with suspected MRSA, with subsequent switching to oral lefamulin and oral moxifloxacin [Figure 2]. ${ }^{35,37,38}$ The results of the study showed that lefamulin was safe and well-tolerated..$^{37,38}$ It was found to be non-inferior to moxifloxacin in the early clinical response (ECR), namely, 96 \pm 24 hour after the first dose of the study drug in the intent-to-treat (ITT) population that was FDA's primary endpoint ( $87.3 \%$ vs. $90.2 \%$, respectively). ${ }^{37,38}$ In addition, it was non-inferior to moxifloxacin in the European Medicines Agency (EMA)'s co-primary endpoints, i.e., the investigator assessment of clinical response (IACR) 5-10 days after last dose of the study drug in the modified ITT (mITT) population $(81.7 \%$ vs. $84.2 \%$, respectively) and in the clinically evaluable (CE) population ( $86.9 \%$ vs. $89.4 \%$, respectively). ${ }^{37,38}$ [Figure 3] LEAP2 (NCT02813694) was another multicenter, randomized, double-blind study that compared the safety and efficacy of oral lefamulin (dose of $600 \mathrm{mg}$ every $12 \mathrm{~h}$ for 5 days) with that of moxifloxacin (dose of $400 \mathrm{mg}$ every 24 hour for 7 days) in patients with CABP and PORT risk class between II and IV. ${ }^{36,39}$ This study aimed to complement the results of the LEAP1 study. ${ }^{36,39}$ It was reported that lefamulin was non-inferior to moxifloxacin regardless of the PORT risk class for both FDA's and EMA's endpoints, i.e., for ECR in the ITT population which was the FDA's primary endpoint (for PORT II, III, IV: $91.8 \%, 91.0 \%$, and $85.0 \%$ for lefamulin; $93.1 \%$, $90.2 \%$, and $85.7 \%$ for moxifloxacin, respectively), and for the EMA's co-primary endpoints of IACR in mITT population $(87.5 \%$ vs. $89.1 \%$, respectively) and in CEtest-of-cure population ( $89.7 \%$ vs. $93.1 \%$, respectively). ${ }^{39}$

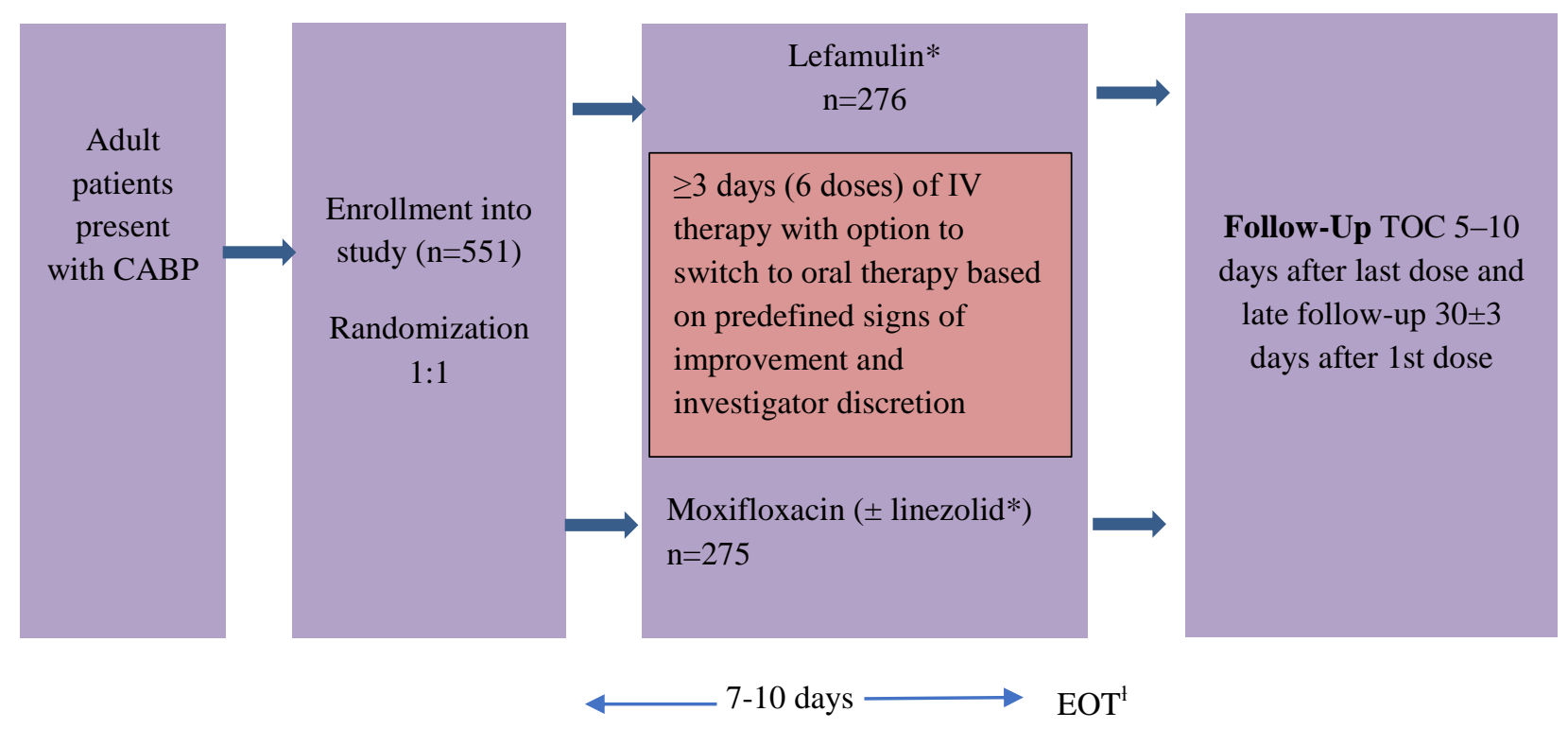

FDA primary endpoint

Early clinical response (ECR)

(Accessed 72-120 hours after first dose)

Improvement in $\geq 2$ of 4 CABP signs/symptoms, ${ }^{*}$ no worsening in any sign/symptom, alive, did not receive nonstudy antibacterial for CABP, ITT population, $12.5 \%$ non-inferiority margin

\section{EMA primary endpoint}

Investigator assessment of clinical response (IACR) (Accessed 5-10 days after last dose)

Improvement or resolution in CABP signs or symptoms such that no additional antibacterial therapy was required to treat CABP, mITT and CE-TOC populations, $10 \%$ non-inferiority margin

Figure 2: Study design of LEAP 1 trial. $^{35}$

$\mathrm{CABP}=$ community-acquired bacterial pneumonia; $\mathrm{CE}-\mathrm{TOC}=$ clinically evaluable at TOC; EMA=European Medicines Agency; EOT=End of treatment; FDA=US Food and Drug Administration; ITT=Intent to treat; IV=Intravenous; mITT= Modified ITT; MRSA=methicillin-resistant Staphylococcus aureus; TOC=test of cure. *If MRSA was suspected, linezolid or placebo was added to moxifloxacin or lefamulin therapy, respectively; if MRSA was confirmed, treatment duration was 10 days; $\dagger$ : EOT assessment was within 2 days after the last dose of study drug; $\$$ : Dyspnea, cough, production of purulent sputum, chest pain. 
Table 1: Summary of lefamulin susceptibility against aerobic pathogens. ${ }^{15,17}$

\begin{tabular}{|c|c|c|c|c|c|c|c|c|}
\hline \multirow{2}{*}{ Gram positive organisms } & \multicolumn{2}{|c|}{$\begin{array}{l}\text { Lefamulin } \\
(\mu \mathrm{g} / \mathrm{ml})\end{array}$} & \multicolumn{2}{|c|}{ Linezolid $(\mu \mathrm{g} / \mathrm{ml})$} & \multicolumn{2}{|c|}{$\begin{array}{l}\text { Vancomycin } \\
(\mu \mathrm{g} / \mathrm{ml})\end{array}$} & \multicolumn{2}{|c|}{$\begin{array}{l}\text { Daptomycin } \\
(\mu \mathrm{g} / \mathrm{ml})\end{array}$} \\
\hline & $\mathrm{MIC}_{50}$ & $\mathrm{MIC}_{90}$ & $\mathrm{MIC}_{50}$ & $\mathrm{MIC}_{90}$ & $\mathrm{MIC}_{50}$ & $\mathrm{MIC}_{90}$ & $\begin{array}{l}\text { MIC } \\
50\end{array}$ & $\mathrm{MIC}_{90}$ \\
\hline Staphylococcus aureus $(\mathrm{n}=5527)$ & 0.12 & 0.12 & 1 & 1 & 1 & 1 & 0.25 & 0.5 \\
\hline MRSA & 0.12 & 0.25 & 1 & 1 & 1 & 1 & 0.25 & 0.5 \\
\hline $\begin{array}{l}\text { Beta hemolytic Streptococcus } \\
(\mathrm{n}=763)\end{array}$ & 0.03 & 0.03 & 1 & 1 & 0.25 & 0.5 & $\begin{array}{l}<=0 . \\
06\end{array}$ & 0.25 \\
\hline $\begin{array}{l}\text { Streptococcus pyogenes, } \\
\text { Group A Streptococci }(n=267)\end{array}$ & 0.03 & 0.03 & 1 & 1 & 0.25 & 0.5 & $\begin{array}{l}<=0 \\
06\end{array}$ & $\begin{array}{l}<=0.0 \\
6\end{array}$ \\
\hline $\begin{array}{l}\text { Streptococcus agalactiae, } \\
\text { Group B Streptococci }(n=334)\end{array}$ & 0.03 & 0.03 & 1 & 1 & 0.5 & 0.5 & 0.12 & 0.25 \\
\hline $\begin{array}{l}\text { Viridans group Streptococcus } \\
(\mathrm{n}=\mathbf{2 4 5})\end{array}$ & 0.12 & 0.5 & 1 & 1 & 0.5 & 0.5 & 0.25 & 0.5 \\
\hline Enterococcus faecium $(n=536)$ & 0.12 & 4 & 1 & 1 & $>16$ & $>16$ & 2 & 2 \\
\hline $\begin{array}{l}\text { Streptococcus pneumoniae } \\
(\mathrm{n}=1473)\end{array}$ & 0.12 & 0.25 & 1 & 1 & 0.25 & 0.5 & - & - \\
\hline Gram negative organism & \multicolumn{2}{|c|}{ Lefamulin } & \multicolumn{2}{|c|}{ Azithromycin } & \multicolumn{2}{|c|}{ Ceftriaxone } & \multicolumn{2}{|c|}{ Moxifloxacin } \\
\hline Haemophilus influenzae $(\mathrm{n}=360)$ & 1 & 2 & 1 & 2 & $<=0.06$ & $<=0.06$ & $<=0.5$ & $<=0.5$ \\
\hline Moraxella catarrhalis $(\mathrm{n}=\mathbf{2 5 3})$ & 0.12 & 0.25 & $<=0.25$ & $<=0.25$ & 0.25 & 0.5 & $<=0.5$ & $<=0.5$ \\
\hline Atypical organisms & \multicolumn{2}{|c|}{ Lefamulin } & \multicolumn{2}{|c|}{ Azithromycin } & \multicolumn{2}{|c|}{ Doxycycline } & \multicolumn{2}{|c|}{ Moxifloxacin } \\
\hline Legionella pneumophila $(\mathrm{n}=30)$ & 0.12 & 0.5 & 0.06 & 0.12 & - & - & 0.06 & 0.12 \\
\hline Chlamydophila pneumonia $(\mathrm{n}=50)$ & 0.02 & 0.04 & - & - & - & - & - & - \\
\hline Mycoplasma pneumonia $(\mathrm{n}=50)$ & 0.006 & 0.006 & - & - & - & - & - & - \\
\hline
\end{tabular}

MIC: Minimum inhibitory concentration; MRSA: Methicillin resistant staphylococcus aureus.

Table 2: TEAEs $>2 \%$ for study medication: safety population in LEAP 1 trial. $^{35,36}$

\begin{tabular}{|lll|}
\hline \multirow{2}{*}{ Adverse effect } & Lefamulin $(\mathbf{n = 2 7 3 )}$ & Moxifloxacin $( \pm$ linezolid) $(\mathbf{n = 2 7 3 )}$ \\
\hline Hypokalemia & $\mathbf{N}(\mathbf{\%})$ & $\mathbf{N}(\boldsymbol{\%})$ \\
\hline Nausea & $8(2.9)$ & $6(2.2)$ \\
\hline Insomnia & $8(2.9)$ & $6(2.2)$ \\
\hline Infusion site pain & $8(2.9)$ & $5(1.8)$ \\
\hline Infusion site phlebitis & $8(2.9)$ & $0(0.0)$ \\
\hline ALT increase & $6(2.2)$ & $3(1.1)$ \\
\hline Hypertension & $5(1.8)$ & $6(2.2)$ \\
\hline Diarrhoea & $2(0.7)$ & $6(2.2)$ \\
\hline
\end{tabular}

LEAP 1: Lefamulin evaluation against pneumonia 1; TEAE: Treatment-emergent adverse event; ALT: Alanine transaminase.

Table 3: Maximum post-dose QTc changes- day 3, n (\%) in LEAP 1 trial. ${ }^{35,36}$

\begin{tabular}{|lll|}
\hline Parameter & Lefamulin & Moxifloxacin ( \pm linezolid) \\
\hline Post dose increase $\mathbf{3 0 - 6 0} \mathbf{~ m s}$ & $12(4.6)$ & $14(5.4)$ \\
\hline Post dose increase $\mathbf{> 6 0} \mathbf{~ m s}$ & $0(0.0)$ & $1(0.4)$ \\
\hline Post dose increase $>\mathbf{5 0 0} \mathbf{~ m s}$ & $1(4.4)$ & $1(0.4)$ \\
\hline
\end{tabular}

QTc-QT corrected interval; LEAP 1: Lefamulin evaluation against pneumonia 1.

Serious adverse events did not dier between the two groups, while the rate of discontinuation was low and similar in both groups. ${ }^{39}$

In conclusion, lefamulin was non-inferior to moxifloxacin for the primary efficacy endpoints and was generally safe and well tolerated.

\section{SAFETY AND ADVERSE DRUG EFFECTS}

The commonest adverse drug reactions reported from both LEAP 1 and LEAP 2 trials include injection site reactions, hepatic enzyme elevation, nausea, hypokalemia, insomnia, and diarrhoea [Table 2]. In the LEAP 1 trial, comparable rates of adverse events were observed in both groups. Discontinuation of study drug or 
withdrawal of a patient from the study due to an adverse event occurred in around $4 \%$ and $2 \%$ in the moxifloxacin and lefamulin groups, respectively. No cases of Clostridium difficile infection were reported in either treatment group. Diarrhoea was observed in $0.7 \%$ and $7.7 \%$ of patients receiving lefamulin and moxifloxacin $( \pm$ linezolid), respectively. Low incidence of liver enzyme elevation was noted in both treatment groups consistent with CABP patient population. The rare but significant cardiovascular adverse event include changes in QT interval of potential clinical concern were also studied but found to be similar frequency between both treatment groups [Table 3]. ${ }^{35-39}$

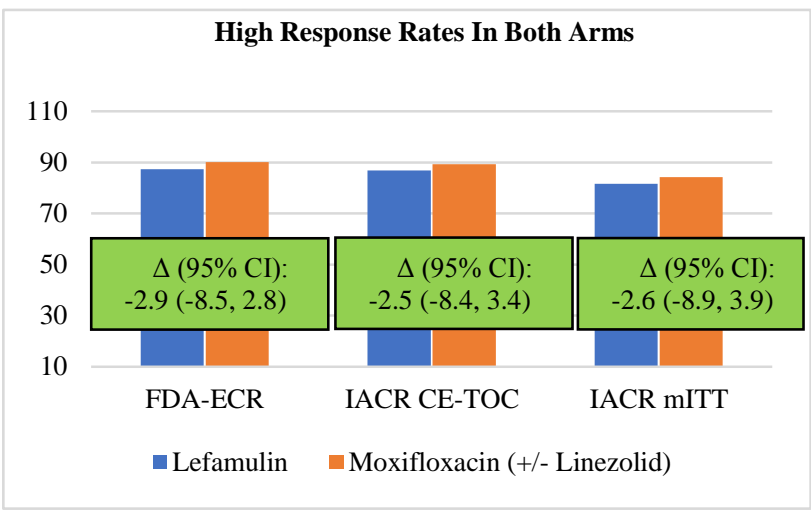

Figure 3: LEAP 1 trial efficacy results- lefamulin met both FDA and EMA primary endpoints. ${ }^{37,38}$

$\mathrm{CE}=$ Clinically evaluable; $\mathrm{ECR}=$ Early clinical response; FDA=US Food and Drug Administration; IACR=Investigator assessment of clinical response; $\mathrm{mITT}=$ Modified intent to treat; TOC $=$ Test of cure.

\section{CONCLUSION, PLACE IN THERAPY}

Judicious stewardship and preferment of appropriate use of novel antimicrobial agents has become necessary due to the rapid emergence of antimicrobial resistance and limiting drug-cost budgets. As more options are being discovered, it eventually boils down on the clinicians to face the challenge of determining the practical role in therapy for these new drugs. The right and responsible decision should be made on the basis of objective parameters including organism susceptibility to the new agent, associated cross-resistance of older agents, site of infection, drug penetration to the tissue being targeted, safety along with drug-drug interactions, cost, and further collateral damage to the general microbiome.

Lefamulin appears to be a promising novel pleuromutilin antibiotic with a wide variety of potential indications for both common and difficult-to-treat infections. Depending on risk stratification, current guidelines recommend use of a PO macrolide or PO fluoroquinolone for outpatient management of CABP. The ability of lefamulin to highly concentrate in lung tissue has been brought about in two phase 3 clinical trials involving CABP (i.e., LEAP 1 and 2 ), one of which suggests lefamulin is non-inferior to moxifloxacin with or without linezolid at ECR.
Lefamulin promises to be an alternative oral agent with festablished activity against new resistant organisms prevalent in the community including macrolide-resistant $M$. pneumoniae and drug-resistant S. pneumoniae. Also, the drug avoids fluoroquinolone antibiotics and the adverse effects associated with their use, as noted in a recent FDA boxed warning update.

Lefamulin is available in both IV and oral formulation making it as an excellent tool to be used in step down therapy (IV to oral), thereby facilitating early hospital discharge. However, further investigation of lefamulin as a first-line agent in hospitalized CABP patients is still warranted. The unique mechanism of action also ensures that the drug activity is not influenced by resistance to other antibacterial classes. However, as the drug has a favourable spectrum of activity, is safe and effective, and is available as an IV and PO formulation, the extensive utility and potential for misuse is high. Long-term studies and post marketing data will be critical in assessing the real benefits and risks of this promising compound.

Funding: No funding sources

Conflict of interest: None declared

Ethical approval: Not required

\section{REFERENCES}

1. Spellberg B, Bartlett J, Wunderink R, et al. Novel approaches are needed to develop tomorrow's antibacterial therapies. Am J Respir Crit Care Med. 2015;191(2):135-40.

2. Conly J, Johnston B. Where are all the new antibiotics? The new antibiotic paradox. Can J Infect Dis Med Microbiol. 2005;16(3):159-60.

3. Fernandes P, Martens E. Antibiotics in late clinical development. Biochem Pharmacol. 2017;133:152-63.

4. Antibiotic resistance threats in the United States, 2013. Centers for Disease Control and Prevention, 2013. Available at: https://www.cdc.gov/drug resistance/threat-report-2013/pdf/ar-threats-2013508.pdf. Accessed on 1 February 2018.

5. Broulette J, Yu H, Pyenson B, Iwasaki K, Sato R. The incidence rate and economic burden of community-acquired pneumonia in a working-age population. Am Health Drug Benefits. 2013;6(8):494.

6. Gibson GJ, Loddenkemper R, Sibille Y, Lundbäck B. European lung white book. Europ Respirator Societ. 2013.

7. File TM, Marrie TJ. Burden of community-acquired pneumonia in North American adults. Postgraduate medicine. 2010;122(2):130-41.

8. Musher DM, Thorner AR. Community-acquired pneumonia. New England Journal of Medicine. 2014;371(17):1619-28.

9. Centres for Disease Control and Prevention (US). Antibiotic resistance threats in the United States, 2013. Centres for Disease Control and Prevention, US Department of Health and Human Services; 2013. 
10. Shehab N, Patel PR, Srinivasan A, Budnitz DS. Emergency department visits for antibiotic-associated adverse events. Clin Infect Dis. 2008;47(6):735-43.

11. US Food and Drug Administration. FDA Drug Safety Communication: FDA updates warnings for oral and injectable fluoroquinolone antibiotics due to disabling side effects. Available at: https:// www.fda.gov/Drugs/DrugSafety/ucm511530.htm. Accessed on 23 March 2018.

12. Brown KA, Khanafer N, Daneman N, Fisman DN. Meta-analysis of antibiotics and the risk of community-associated Clostridium difficile infection. Antimicrob Agents Chemotherap. 2013;57(5):232632.

13. Eyal Z, Matzov D, Krupkin M, Paukner S, Riedl R, Rozenberg H, Zimmerman E, et al. A novel pleuromutilin antibacterial compound, its binding mode and selectivity mechanism. Sci Rep. 2016;6:39004.

14. Mendes RE, Farrell DJ, Flamm RK, Talbot GH, Ivezic-Schoenfeld $\mathrm{Z}$, Paukner $\mathrm{S}$, et al. In vitro activity of lefamulin tested against Streptococcus pneumoniae with defined serotypes, including multidrug-resistant isolates causing lower respiratory tract infections in the United States. Antimicrob Agents Chemother. 2016;60:4407-11.

15. Sader HS, Paukner S, Ivezic-Schoenfeld Z, Biedenbach DJ, Schmitz FJ, Jones RN. Antimicrobial activity of the novel pleuromutilin antibiotic BC3781 against organisms responsible for communityacquired respiratory tract infections (CARTIs). J Antimicrob Chemother. 2012;67:1170-5.

16. Waites KB, Crabb DM, Duffy LB, Jensen JS, Liu Y, Paukner S. In vitro activities of lefamulin and other antimicrobial agents against macrolide-susceptible and macrolide-resistant Mycoplasma pneumoniae from the United States, Europe, and China. Antimicrob Agents Chemother. 2017;61:e02008-16.

17. Paukner S, Sader HS, Ivezic-Schoenfeld Z, Jones RN. Antimicrobial activity of the pleuromutilin antibiotic BC-3781 against bacterial pathogens isolated in the SENTRY antimicrobial surveillance program in 2010. Antimicrob Agents Chemother. 2013;57:4489-95.

18. Paukner S, Gruss A, Fritsche TR, Jones RN. In vitro activity of the novel pleuromutilin BC-3781 tested against bacterial pathogens causing sexually transmitted diseases (STD). 53rd Interscience Conference on Antimicrob Agents Chemotherap. 2013; Abstract e-1183.

19. Novak R, Shlaes DM. The pleuromutilin antibiotics: a new class for human use. Curr Op Invest Drugs. 2010;11(2):182-91.

20. Eyal Z, Matzov D, Krupkin M, Wekselman I, Paukner S, Zimmerman E, et al. Structural insights into species-specific features of the ribosome from the pathogen Staphylococcus aureus. Proc Natl Acad Sci USA. 2015;112(43):E5805-14.
21. Paukner S, Riedl R. Pleuromutilins: potent drugs for resistant bugs- mode of action and resistance. Cold Spring Harb Perspect Med. 2017;7:a027110.

22. Novak R. Are pleuromutilin antibiotics finally fit for human use?. Ann N.Y. Acad Sci. 2011;1241:71-81.

23. Sader HS, Biedenbach DJ, Paukner S, et al. Antimicrobial activity of the investigational pleuromutilin compound BC-3781 tested against Gram-positive organisms commonly associated with acute bacterial skin and skin structure infections. Antimicrob Agents Chemother. 2012;56(3):1619-23.

24. Mendes, R, Farrell, D, Flamm R, et al. Antimicrobial potency of lefamulin tested against Streptococcus pneumoniae with defined serotypes, including multidrug-resistant isolates causing lower respiratory tract infections in the United States. Antimicrob Agents Chemother 2016; 60(7):4407-11

25. Ross JE, Sader HS, Ivezic-Schoenfeld Z, Paukner S, Jones RN. Disk diffusion and MIC quality control ranges for $\mathrm{BC}-3205$ and $\mathrm{BC}-3781$, two novel pleuromutilin antibiotics. J Clin Microbiol. 2012;50(10):3361-4.

26. Craig WA, Andes D, Ivezic-Schoenfeld Z, Wicha WW, Novak R. In vivo pharmacodynamic activity of BC-3781. 20th European Congress of Clinical Microbiology and Infectious Diseases 2010; Abstract P907.

27. Rodvold KA. Introduction: lefamulin and pharmacokinetic/pharmacodynamic rationale to support the dose selection of lefamulin. J Antimicrobial Chemotherap. 2019;74(Suppl 3):iii2-4.

28. Wicha WW, Lell C, Strickmann DB, Heilmayer W, Schoenfeld ZI, Prince WT. Safety, tolerability and pharmacokinetics of orally administered BC-3781, a novel antimicrobial. 53rd Interscience Conference on Antimicrobial Agents and Chemotherapy 2013; Abstract A-013.

29. Wicha WW, Leil C, Seltzer E, Prince WT, Gelone SP. Pharmacokinetics and safety of an oral, immediate-release (IR) tablet formulation of lefamulin in fed and fasted healthy subjects. 27th European Congress of Clinical Microbiology and Infectious Diseases 2017; Abstract P1336.

30. Zeitlinger M, Schwameis, R, Burian A, Burian B, Matzneller P, Müller M, et al. Simultaneous assessment of the pharmacokinetics of a pleuromutilin, lefamulin, in plasma, soft tissues, and pulmonary epithelial lining fluid. J Antimicrob Chemother. 2016;71(4):1022-6.

31. Schmidt U, Wicha WW, Obermayr F. BC-3781: evaluation of the CYP3A interaction potential. 21st European Congress of Clinical Microbiology and Infectious Diseases 2011; Abstract P1524.

32. Label (PDF)-FDA. XENLETA (Lefamulin). Highlights of prescribing information. Available at: https://www.accessdata.fda.gov/drugsatfda_docs/labe 1/2019/211672s000,211673s000lbl.pdf Accessed on 16 September 2019.

33. Wicha WW, Prince WT, Lell C, Heilmayer W, Gelone SP. Pharmacokinetics and tolerability of lefamulin following intravenous and oral dosing. J 
Antimicrobial Chemotherap. 2019;74(Suppl 3):iii1926.

34. Koulenti D, Xu E, Mok IYS, Song A, Karageorgopoulos DE, Armaganidis A, et al. Comment on "Novel Antibiotics for MultidrugResistant Gram-Positive Microorganisms. Microorganisms, 2019, 7, 270". Microorganisms. 2019;7(10):386.

35. Study to compare lefamulin to moxifloxacin (with or without linezolid) for the treatment of adults with pneumonia (LEAP). Available at: https://clinicaltrials.gov/ct2/show/NCT02559310. Accessed on 15 September 2019.

36. Study to compare lefamulin to moxifloxacin for the treatment of adults with pneumonia (LEAP2). Available at: https://clinicaltrials.gov/ct2/show/NCT 02813694 . Accessed on 15 September 2019.

37. File T, Goldberg L, Paukner S, Das A, Gelone SP, Saviski J, et al. Efficacy of lefamulin (LEF) vs. moxifloxacin (MOX) Against common pathogens in adults with community-acquired bacterial pneumonia
(CABP): results from the phase 3 lefamulin evaluation against pneumonia (LEAP 1) study. Open Forum Infect. Dis. 2018;5(S1):S711-2.

38. File TM, Goldberg L, Das A, Sweeney C, Saviski J, Gelone SP, et al. Efficacy and Safety of IV-to-Oral Lefamulin, a Pleuromutilin Antibiotic, for Treatment of Community-Acquired Bacterial Pneumonia: The Phase 3 LEAP 1 Trial Clin Infect Dis. 2019;4.

39. Alexander E, Goldberg L, Das A, Moran GJ, Sandrock C, Gasink LB, et al. LB6. Oral lefamulin is safe and effective in the treatment of adults with community-acquired bacterial pneumonia (CABP): results of lefamulin evaluation against pneumonia (LEAP 2) study. Open Forum Infect Dis. 2018;5(S1):S761.

Cite this article as: Dubey AP, Maggo S, Karan A, Singh NK, Bhaskaran S. Lefamulin- novel pleuromutilin drug for community acquired bacterial pneumonia. Int J Basic Clin Pharmacol 2019;8:278390. 